\title{
Double in Situ Hybridization in Combination with Digital Image Analysis: A New Approach to Study Interphase Chromosome Topography
}

\author{
PATRICIA EMMERICH, ${ }^{*, 1}$ PETER LOOS, ${ }^{\dagger}$ ANNA JAUCH, ${ }^{*}$ \\ ANTON H. N. HOPMAN, $\ddagger$ JOOP WIEGANT, MICHAEL J. HIGGINS" \\ BRADLEY N. WHITE, MELS VAN DER PLOEG, $\S$ \\ CHRISTOPH CREMER, ${ }^{\dagger}$ and THOMAS CREMER*
}

*Institute of Anthropology and Human Genetics, University of Heidelberg, INF 328, D-6900 Heidelberg, Federal Republic of Germany; ${ }^{\dagger}$ Institut of Applied Physics I, University of Heidelberg, D-6900 Heidelberg, Federal Republic of Germany; $\ddagger$ Institute of Pathology, University of Nijmegen, NL-6525 PB Nijmegen, The Netherlands; $\$$ Department of Cytochemistry and Cytometry, Sylvius Laboratories, State University, NL-2333 AL Leiden, The Netherlands; and "Department of Biology, Queens University, Kingston, Ontario, Canada, K72 3 N6

Double in situ hybridization with mercurated and biotinylated chromosome specific DNA probes in combination with digital image analysis provides a new approach to compare the distribution of homologous and nonhomologous chromosome targets within individual interphase nuclei. Here we have used two DNA probes representing tandemly repeated sequences specific for the constitutive heterochromatin of the human chromosomes 1 and 15, respectively, and studied the relative arrangements of these chromosome targets in interphase nuclei of human lymphocytes, amniotic fluid cells, and fibroblasts, cultivated in vitro. We have developed a $2 \mathrm{D}$-image analysis approach which allows the rapid evaluation of large numbers of interphase nuclei. Models to test for a random versus nonrandom distribution of chromosome segments are discussed taking into account the three-dimensional origin of the evaluated $2 \mathrm{D}$-distribution. In all three human diploid cell types the measurements of target-target and target-center distances in the 2D-nuclear image revealed that the labeled segments of the two chromosomes 15 were distributed both significantly closer to each other and closer to the center of the nuclear image than the labeled chromosome 1 segments. This result can be explained by the association of nucleolus organizer regions on the short arm of chromosome 15 with nucleoli located more centrally in these nuclei and does not provide evidence for a homologous association per se. In contrast, evaluation of the interphase positioning of the two chromosome 1 segments fits the random expectation in amniotic fluid and fibroblast cells, while in experiments using lymphocytes a slight excess of larger distances between these homologous targets was occasionally observed. 2D-distances between the labeled chromosome 1 and 15 segments showed a large variability in their relative positioning. In conclusion our data do not support the idea of a strict and permanent association of these homologous and nonhomologous targets in the cell types studied so far. (C) 1989 Academic Press, Inc.

Cell type specific chromatin arrangements in interphase nuclei may reflect processes of cellular differentiation and cell specific gene expression [1-4].

So far the methodology has been lacking to put this hypothesis to rigorous

1 To whom reprint requests should be addressed. 
tests. Such a methodology can be provided by double in situ hybridization with chemically modified chromosome specific DNA probes combined with image analysis. This approach makes it possible to study the relative arrangements of both homologous and heterologous chromosome regions directly in the interphase nucleus. In this paper we describe and discuss this new approach in detail, using probes which (under stringent conditions) hybridize specifically to the constitutive heterochromatin of the human chromosomes 1 and 15 [5-7]. Experiments were carried out with human fibroblasts, amniotic fluid cells, and lymphocytes before and after stimulation with phytohemagglutinin (PHA). We have chosen these probes for several reasons. A nonrandom distribution has consistently been observed for the acrocentric chromosomes 15 in analyses of human metaphase plates. This chromosome plays a role in the formation of the nucleolus and is often associated with the other acrocentric human chromosomes 13, 14, 21, and 22, which also bear nucleolus organizer regions (NORs). Accordingly, we expect for nuclei with more centrally located nucleoli that the centromeric region of chromosome 15, which is close to the NOR on its short arm, should also be located more centrally. Thus specific staining of the centromeric heterochromatin of this chromosome should provide a valuable test for the validity of our approach. In contrast, conflicting results have been reported regarding the interphase and metaphase distribution of chromosome 1 in different cell types. Discrepancies of published data concern the question of homologous association [8-10], the preferential localization of this chromosome either in the center or in the periphery of metaphase plates and interphase nuclei, respectively [11-14], and in particular, the possible association of the centromeric region of chromosome 1 with the NOR-bearing short arms of acrocentric chromosomes at metaphase [15] and with the nucleolus at interphase [16].

In our study we address some of the puzzling contradictions in the findings of different authors which are exemplary for investigations of chromosome topography in general.

\section{MATERIAL AND METHODS}

\section{Cultivation and Preparation of Cells}

(1) Lymphocytes. Nonstimulated lymphocytes were isolated from healthy female and male persons, using Lymphoprep (Nyegaard \& Co., Oslo) according to the manufacturer's instructions. Cells were fixed with methanol/acetic acid $(3: 1, \mathrm{v} / \mathrm{v})$ and dropped on slides precleaned with absolute ethanol. Lymphocytes from peripheral blood were cultivated in chromosome-medium B, containing PHA (Seromed, Berlin). After $72 \mathrm{~h}$ of cultivation in a humified incubator with $5 \% \mathrm{CO}_{2}$ at $37^{\circ} \mathrm{C}$, cells were fixed with methanol/acetic acid either directly or after an additional $2 \mathrm{~h}$ exposure to colcemid (Boehringer-Mannheim, $0.5 \mu \mathrm{g} / \mathrm{ml}$ ) and hypotonic treatment $\left(0.075 \mathrm{M} \mathrm{KCl}\right.$ for $25 \mathrm{~min}$ at $37^{\circ} \mathrm{C}$ ).

(2) Amniotic fluid cells and fibroblasts. Amniotic fluid cells (F-type) [17] from several pregnancies, with male and female fetuses, and human fibroblasts (Flow 2000), in phase II of their replicative lifespan in vitro, were cultivated as described above in Ham's F-10 medium supplemented with $20 \%$ fetal calf serum (FCS), $10 \mu \mathrm{g} / \mathrm{ml}$ streptomycin, $10 \mathrm{IU} / \mathrm{ml}$ penicillin, and sodium bicarbonate (1.2 g/liter). Cells were detached using $5 \mathrm{ml}$ trypsin (0.05\%)/EDTA (0.02\%) (Boehringer-Mannheim) and either fixed immediately with methanol/acetic acid $(3: 1)$ or cultured again on sterile slides in petri dishes at $37^{\circ} \mathrm{C}$ in Ham's F-10 medium overnight. In the latter case, slides were washed with PM 16 buffer (Serva, Heidelberg) before they were fixed by slowly adding fixative to the buffer. 


\section{Emmerich et al.}

\section{DNA Probes and Labeling Procedures}

pUC 1.77 designates a clone of the plasmid vector pUC9 containing a 1.77-kb-long human EcoRI fragment as insert. The insert was isolated from the human satellite DNA fraction II/III and represents a tandemly organized repetitive sequence in the region 1q12 [5]. D15Z1 is a DNA sequence which is predominantly located in the constitutive heterochromatin of chromosome 15 [6]. A 1.8-kb Sau3A tragment of the sequence, which is organized in long tandem arrays with a copy number of 3000 per haploid genome was cloned in the plasmid vector pUC8. Plasmid DNAs containing these inserts were purified [18] and were either nick-translated with biotin-11-dUTP [19] or mercurated by incubation with mercury acetate $[20,21]$. In the following we refer to pUC 1.77 as probe $1 \mathrm{c}$ and to the plasmid containing the 1.8 -kb Sau $3 \mathrm{~A}$ fragment as probe $15 \mathrm{c}$.

\section{In Situ Hybridization Conditions}

(1) In situ hybridization with biotinylated DNA probes. In situ hybridizations with the biotinylated DNA probes 1c and 15c were carried out as described by Schardin et al. [22] with the following modifications. We used $2 \mu \mathrm{l}$ of the hybridization mixture per square centimeter of the slide. The final DNA concentration was approx $1 \mu \mathrm{g} / \mathrm{ml}$ in a hybridization mixture, containing $65 \%$ formamide, $2 \times$ SSC. Under these conditions hybridization with probe $15 \mathrm{c}$ resulted in strong signals exclusively over the centromeric region of chromosome 15 . At lower concentrations of formamide $(<65 \%)$, additional labeling was observed over the constitutive heterochromatin of other chromosomes, especially chromosome 9 [6]. In case of probe 1c, $60 \%$ formamide was sufficient to yield strong signals exclusively on chromosome 1 [7]. The hybridization mixture was added to the slides, sealed with a coverglass, and cellular and probe DNA was denatured simultaneously at $72^{\circ} \mathrm{C}$ for $10 \mathrm{~min}$. Hybridization was performed overnight in a wet chamber at $42^{\circ} \mathrm{C}$. The slides were washed twice for 30 min each in $50 \%$ formamide/ $\times$ SSC (SSC: $150 \mathrm{mM}$ sodium chloride, $15 \mathrm{mM}$ sodium citrate, $\mathrm{pH} 7.0$ ) at $37^{\circ} \mathrm{C}$ for $30 \mathrm{~min}$ and in $0.1 \times \mathrm{SSC}$ at $37^{\circ} \mathrm{C}$.

(2) In situ hybridization with mercurated DNA probes. In situ hybridization experiments with mercurated DNA probes were carried out as described by Hopman et al. [23] with modifications as described above for biotinylated probes.

(3) Simultaneous in situ hybridization with two DNA probes. For simultaneous hybridization with one mercurated and one biotinylated DNA probe, both probes were used at approx $1 \mu \mathrm{g} / \mathrm{ml}$ in a hybridization mixture as described above with $65 \%$ formamide. Conditions of hybridization and washing procedures were the same as described above for mercurated probes.

\section{Detection of Hybridized DNA Probes}

Hybridization sites of biotinylated DNA probes were visualized with an alkaline phosphatase reaction using the BRL DNA-Detection System No. 8293 A [22], with a peroxidase reaction using the DETEK I-hrp Kit (ENZO Ortho Diagnostik, Heidelberg), or with FITC-avidin (DETEK I-fav, ENZO Ortho Diagnostik, Heidelberg). Hybridization sites of mercurated DNA probes were detected with a peroxidase reaction or by indirect immunofluorescence [21]. The detection procedure after simultaneous hybridization with mercurated and biotinylated probes was described in detail by Hopman et al. [24] and Cremer et al. [7].

\section{Evaluation of Nuclei}

In each experiment some 300 nuclei were evaluated from areas with good hybridization efficiency, i.e., some $60 \%$ of the nuclei showed two major "hybridization spots" for each probe in these selected areas [7]. About $40 \%$ of the nuclei were excluded from further evaluation according to the following criteria: (a) nuclei with odd shapes, i.e., shapes which could not conveniently be fitted by an ellipse; (b) nuclei showing considerable background or insufficient hybridization. Nuclei with a single spot, 10-20\%, were generally excluded. They were however included in cases where this single spot was considered to be due to an overlap of two hybridization sites by criteria of its shape, size and intensity (see also Results); (c) Rare nuclei where additional minor hybridization spots could not easily be distinguished on the basis of intensity were also excluded.

Camera lucida drawings were made from nuclei clearly showing the nuclear edge and the position of the hybridization spots. The drawings were then recorded with a BW-videocamera (Bosch; type T1VK9B1) and digitized (256 gray levels). The digitized images were stored in a VAX 11-780 computer which was also used for the following image analysis. The program applied [25] is based on that described by Zinser and Komitowski [26]. It was modified for the application presented here. 
First for each drawing a gray level histogram was established. From this, by a suitable threshold selection, a binary image was produced, and served to identify the nuclear edge. The tablet was also used to indicate the positions of the $1 \mathrm{c}$ and $15 \mathrm{c}$ spots, respectively. The following steps were performed automatically: The center of the nuclear area and the coordinates of each chromosome spot were identified. Furthermore, ellipses were adapted to each nuclear edge, and the lengths of the two main axes were calculated for each ellipse. For a given nucleus, distances between spots (target-target distances) were determined for all possible combinations of homologous and nonhomologous hybridization spots (1c-1c, $15 c-15 c$, and the two possible $1 c-15 c$ distances), as well as between each hybridization spot and the center of the nuclear image (target-center distances).

Double hybridization experiments provide the advantage that target-target or target-center distances obtained for two different probes can be compared directly in each individual nucleus. In case of a similar distribution of the hybridization sites, obtained for both probes, we would expect mean ratios of the respective distances in the evaluated set of nuclei close to 1 , while a clear deviation from 1 would indicate a different distribution. The significance of such a deviation can be tested by Student's $t$ test (see legend of Table 1).

Using the procedures described, the evaluation of the camera lucida drawings of an experiment (typical value, 300 nuclei) lasts about $2-3 \mathrm{~h}$, whereas the completely manual evaluation and calculation of data [27] of such an experiment took several days. It is possible to automatically record the edge of nuclei and the positions of hybridization sites directly from the microscope slide (Figs. $1 a, b$ ). However, this procedure turned out to be more time consuming and did not improve the accuracy of the measurements. Therefore, we have preferred camera lucida drawings as an intermediate step in the evaluation.

\section{Model Calculations}

For comparison, theoretical distribution functions of normalized distances were calculated (Fig. 2). For these "model curves" it was assumed that the three-dimensional shape of the nuclei studied can be reasonably approximated by either ellipsoids or flat elliptical cylinders. For each experiment a set of ellipsoids and cylinders, respectively, was constructed as model nuclei. The distribution of the 2Dmain axes was the same as determined for the experimental nuclei in each given experiment. Notably, the model distribution functions of 2D-distances as described below are independent from the size of

\section{TABLE 1}

Ratio of distances (1c-1c/15c-15c) and (1c-CNI/15c-CNI) in double hybridization experiments with probe $1 \mathrm{c}$ ( $\mathrm{Hg} /$ peroxidase detection) and probe $15 \mathrm{c}$ (biotinlalkaline phosphatase detection)

\begin{tabular}{lccc}
\hline & \multicolumn{3}{c}{ Cell type } \\
\cline { 2 - 4 } & $\begin{array}{c}\text { Stimulated } \\
\text { lymphocytes }\end{array}$ & $\begin{array}{c}\text { Amniotic } \\
\text { fluid cells }\end{array}$ & Fibroblasts \\
\hline Mean ratio & & & \\
(1c-1c/15c-15c) & $1.643^{*}$ & $2.025^{*}$ & $2.959^{*}$ \\
SEM & 0.07 & 0.1 & 0.29 \\
$(1 \mathrm{c}-\mathrm{CNI} / 15 \mathrm{c}-\mathrm{CNI})$ & $1.31^{*}$ & $1.545^{*}$ & $1.698^{*}$ \\
SEM & 0.04 & 0.04 & 0.096 \\
$N$ & 275 & 295 & 120 \\
\hline
\end{tabular}

Note. $N$, number of evaluated nuclei; CNI, center of the nuclear image; SEM, standard error of the mean.

*Significant deviation from a random distribution at the $99 \%$ level, tested by Student's $t$ test. To apply this test the averages of the logarithms of the ratios were calculated. 


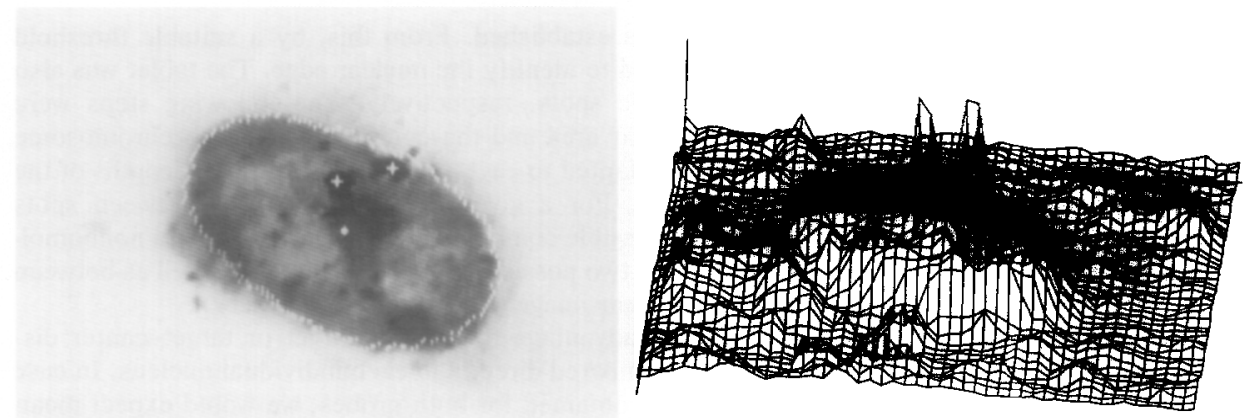

Fig. I. (a) Digitized image of an amniotic fluid cell nucleus after hybridization with probe 1c (biotin/alkaline phosphatase detection) and Giemsa staining. The nuclear edge, the positions of the two hybridization spots, and the center of the nuclear image have been automatically defined. Bar represents $10 \mu \mathrm{m}$. (b) Isometric display of gray values of the digitized image of the same nucleus. The two peaks indicate the two 1c spots. The nuclear edge is also clearly delineated.

the third axis giving the height of the ellipsoid or cylinder. For a given number of ellipsoids or cylinders, respectively, two points were generated by a Monte Carlo procedure. To assure the uniform and independent distribution of the two points, each point was randomly generated for a cubus. Points which did not fit into the respective ellipsoid or cylinder covered by this cubus were eliminated. Normalized distances were calculated after orthogonal projection onto plane A, B (see Fig. 2): (a) between the two locations (target-target distances) and (b) between each location and the center of the resulting ellipse (target-center distances). If not indicated otherwise, these calculations were performed for $N=10,000$ ellipsoids or cylinders (Fig. 2, smooth curves). It was assumed that these model curves reflect the "true" model curves to be expected for samples of unlimited size. In fact, differences between several repetitions of these model curves could not be graphically depicted. For each ccll typc, model curves obtained in different experiments were very similar, indicating that the 2D-axes distributions did not significantly change throughout the course of our experiments. However, while the mean axes proportions obtained for fibroblast and F-type amniotic fluid cell nuclei were identical $(0.62 \pm 0.06$ (SD) vs $0.62 \pm 0.05$ (SD)), the mean axes proportions obtained for lymphocyte nuclei were significantly different $(0.88 \pm 0.01(\mathrm{SD}))$. Accordingly, model curves obtained for lymphocyte nuclei were also significantly different from those obtained for the two other cell types.

To test the fitting of the experimental and the model curves, the Kolmogorov-Smirnov test was used [28]. This test allows for a given level of significance $\alpha$ a maximum of the ordinate deviations of a given experimental function from the model functions. The maximum deviations which can be tolerated for $\alpha=0.01$ are depicted in Fig. 2 by hatched lines. Criteria for a significant deviation of the experimental data from a supposedly random distribution were intentionally defined rather broadly to avoid statistical artifacts due to differences in the actual three-dimensional shape of nuclei from the shapes of model nuclei. Accordingly, such differences were accepted as significant only if the experimental curve crossed the "worst case borderline" (borderline of the ellipsoid distribution for the region left to the model curve, or borderline of the cylinder distribution for the region right to the model curves, respectively). This means that $99 \%$ of model curves generated for a given number of ellipsoid and cylinder model nuclei equal to the number of nuclei actually evaluated in a given experiment (about 300 ) can be statistically expected to fall within the range included by these borderlines. In the following, we refer to this range as range 2 . A small but still significant deviation from a random distribution cannot be excluded for experimental curves found within range 2 . In any case, experimental curves within this range indicate a large variability of the respective positions within the investigated set of nuclei. If experimental curves cross the left borderline (range 1), significantly smaller target-target or target-center distances are indicated. Significantly larger distances are indicated if the experimental curves cross the right borderline (range 3 ). 

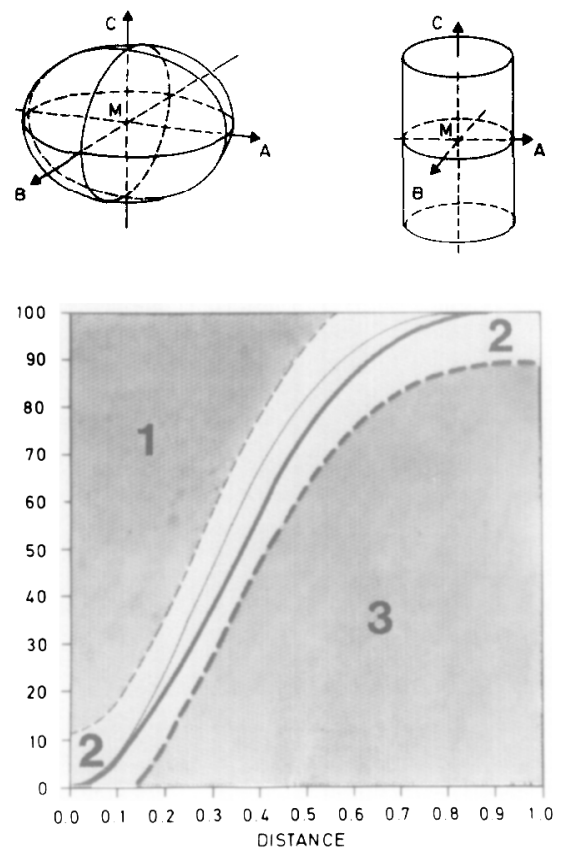

Fig. 2. Model curves showing 2D target-target distances for 10,000 ellipsoids (smooth thin curve) and 10,000 cylinders (smooth thick curve). (Abscissa) Normalized 2D-distance $\beta$ between two points. (Ordinate) Percentage of ellipsoids or cylinders with a normalized distance $\beta$ equal to or smaller than the corresponding $\beta$ shown on the abscissa (accumulated frequency). In each ellipsoid and cylinder, respectively, two points were distributed randomly within the three-dimensional space. 2D-distances were calculated after orthogonal projection of the actual 3D-distance between these points on the ellipse shown in plane A, B. 2D-distances were normalized by setting the major axis of this ellipse at 1. Axis proportions for these model ellipses were the same as the axis proportions obtained for a given set of evaluated nuclei with elliptical shape. The resulting model curves are independent from the height of the ellipsoids and cylinders, respectively. Hatched borderlines indicate $99 \%$ confidence limits for the ellipsoid model (left thin line) and the cylinder model (right thick line), according to the Kolmogorov-Smirnow test (see text). Range 1, Experimental curves obtained for 2D target-target distances (see Figs. $6 a, 7 a, c, e, 8 a-c$ ), which cross the left borderline into range 1 indicate significantly smaller mean $2 \mathrm{D}$-distances than expected in case of a random three-dimensional distribution of the chromosomal targets. Range 2, Experimental curves located entirely within this range, both with regard to target-target and target-center model curves, indicate a highly variable and possibly random distribution of distances. Range 3, Experimental curves which cross the right borderline into range 3 indicate significantly larger distances than expected in case of a random distribution of distances.

\section{RESULTS}

The specificity of the hybridization conditions was tested by double in situ hybridization experiments in normal human lymphocyte metaphase plates, using probes $1 \mathrm{c}$ and $15 \mathrm{c}$. Figure 3 shows examples of double hybridization with biotinylated probe 15c (alkaline phosphatase detection (a) and FITC detection (b)) and mercurated probe 1c (peroxidase detection (a) and TRITC detection (b)). Under the hybridization conditions used, major hybridization signals were confined exclusively to the C-band $1 \mathrm{q} 12$ for probe $1 \mathrm{c}$ (Fig. $3 a$, dark brown spots, Fig. $3 b$, red spots) and to the pericentric region of chromosome 15 for probe $15 \mathrm{c}$ (Fig. $3 a$, blue spots, Fig. $3 b$, green spots). The majority of interphase nuclei (some 

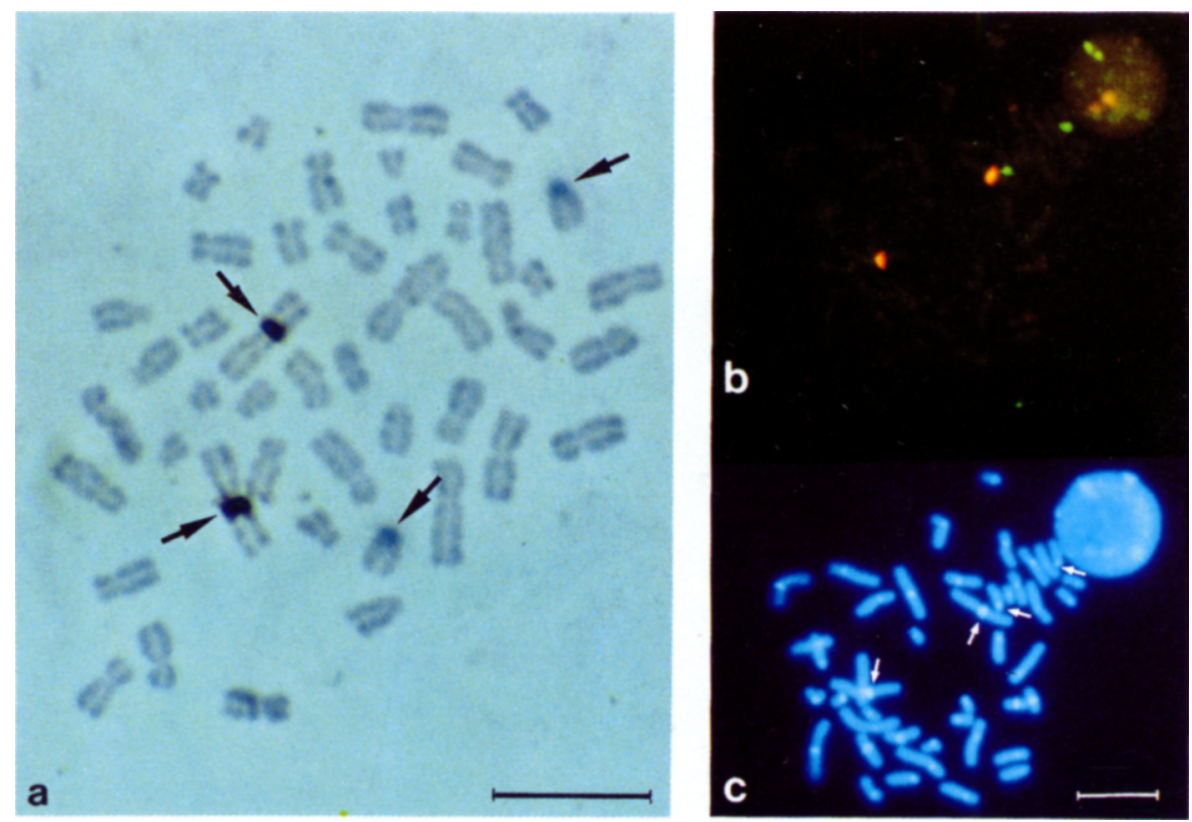

Fig. 3. (a) Metaphase spread from a PHA-stimulated human lymphocyte culture after double hybridization with probe $15 \mathrm{c}$ (biotin/alkaline phosphatase detection) and probe $1 \mathrm{c}(\mathrm{Hg} /$ peroxidase detection) and Giemsa staining. Arrows point to specific label at the centromere regions of both chromosomes 15 (blue) and 1 (brown), indicated by black arrows. (b) Metaphase spread from a PHAstimulated human lymphocyte culture after double hybridization with probe $15 \mathrm{c}$ (biotin/FITC detection, green) and probe $1 \mathrm{c}(\mathrm{Hg} / \mathrm{TRITC}$ detection, red). (c) The same metaphase after DAPI staining. White arrows indicate the constitutive heterochromatic regions of the chromosomes 1 and 15 specifically labeled in (b). Bar represents $10 \mu \mathrm{m}$.
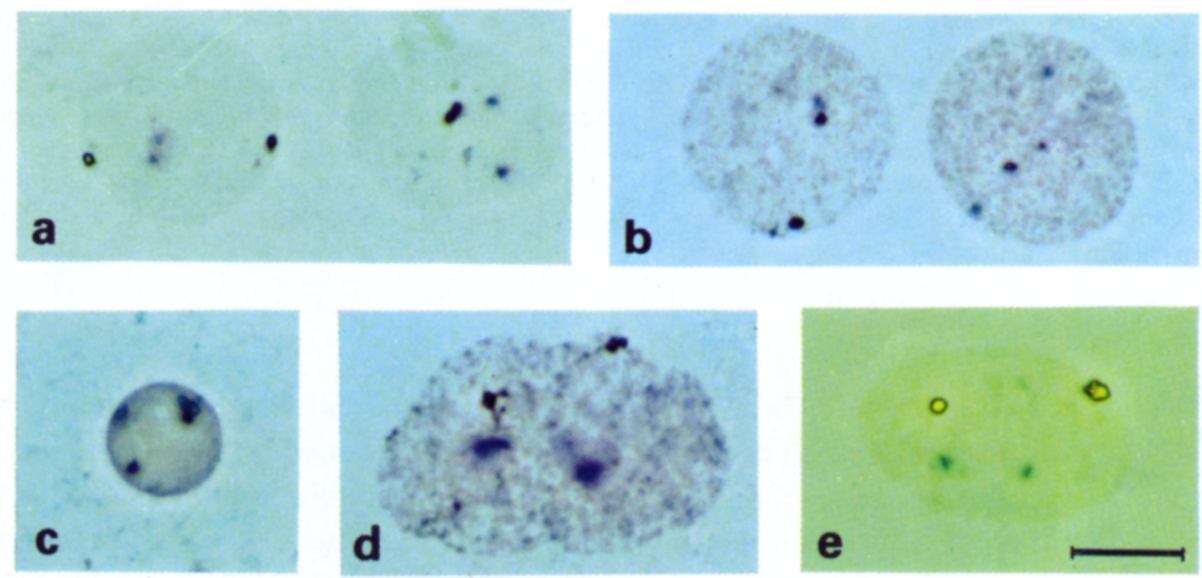

Fig. 4. Human interphase nuclei after double hybridization with the biotinylated probe $15 \mathrm{c}$ (alkaline phosphatase detection, blue signals) and mercurated probe 1c (peroxidase detection, brown signals). Nuclei show various positions of the four hybridization signals. $(a, b)$ Nuclei from a PHA-stimulated normal lymphocyte culture after colcemid and hypotonic treatment. (c) Nucleus from a nonstimulated lymphocyte, fixed without any pretreatment. (d) Nucleus from a fibroblast after fixation in situ. (e) Nucleus from an amniotic fluid cell of a primary culture, detached by trypsin, fixed, and dropped on slides. Nuclei shown in $(a-d)$ are Giemsa stained, the nucleus in $(e)$ was not counterstained and photographed with phase contrast. Bar represents $10 \mu \mathrm{m}$. 
$60 \%$ ) revealed two major hybridization signals for each probe. These nuclei were used for further evaluation (see also Material and Methods).

Figures $4 a-e$ show examples of interphase nuclei from lymphocyte, fibroblast, and amniotic fluid cell cultures after double hybridization with probe $1 \mathrm{c}(\mathrm{Hg} / \mathrm{per}-$ oxidase detection) and probe 15c (biotin/alkaline phosphatase detection). 1c hybridization spots (brown, black arrows) and 15c hybridization spots (blue, white arrows) were easily distinguishable. Obviously, a great variability of 2Ddistances between the homologous (Figs. 4, 5) and heterologous chromosome regions (Fig. 4) can be observed in these nuclei. In Fig. 5 the nucleoli are clearly visible in three amniotic fluid cell nuclei after staining with Giemsa.

We have developed an approach for the rapid statistical evaluation of several hundred nuclei per experiment by 2D-image analysis (see Materials and Methods). This analysis should provide information (a) on the question of homologous association by measurements of distances between two homologous targets, i.e. $1 c-1 c$ or $15 c-15 c$ distances; (b) on the question of a uniform versus nonuniform distribution of a given chromosomal subregion, i.e., $1 \mathrm{c}$ or $15 \mathrm{c}$, within a set of nuclei by measurement of target-center distances (together with data on (a)); (c) on the question of a mutually dependent versus nondependent distribution of nonhomologous targets by measurements of $1 \mathrm{c}-15 \mathrm{c}$ distances. Figures 6 and 7 , described below, show results concerning questions (a) and (b), while Fig. 8 presents data concerning question (c).

Five in situ hybridization experiments were performed with amniotic fluid cells (Fig. 6), two of them were double hybridizations with biotinylated probe $15 \mathrm{c}$ (alkaline phosphatase detection) and mercurated probe 1c (peroxidase detection), three were hybridizations with probe $15 \mathrm{c}$ (biotin/peroxidase detection) only. In order to compare different cell preparations, one double hybridization and one single hybridization was performed with cells fixed immediately after trypsin/ EDTA detachment and thereafter dropped on slides, the other experiments were carried out with preparations of cells grown on slides and fixed in situ. When compared to model curves, all experimental curves for probe $15 \mathrm{c}$ showed significantly smaller target-target and target-center distances. In contrast, experimental curves for probe 1c were localized within range 2 and thus fit our expectation for the random positioning of the two 1c sites (compare Fig. 2). The same results were found in fibroblast nuclei (data not shown).

Figure 7 shows the results of in situ hybridization of lymphocytes with probes $1 \mathrm{c}$ and $15 \mathrm{c}$. We have chosen three different manners of cell pretreatment before fixation (see legend to Fig. 7). These experiments were intended to provide information both on whether noncycling lymphocytes at G0 differ from cycling lymphocytes with respect to the arrangements of the investigated chromosome pairs and on effects of colcemid and hypotonic shock. Our data show a pronounced variability of chromosome positioning both for noncycling (Figs. $7 a, b$ ) and cycling lymphocytes (Figs. $7 c-f$ ). Curves obtained for $15 c-15 c$ distances and 15c-center distances were consistently located left from the respective curves obtained for probe 1c in all lymphocyte experiments, although these differences were less pronounced than in fibroblasts and amniotic fluid cell nuclei (compare 


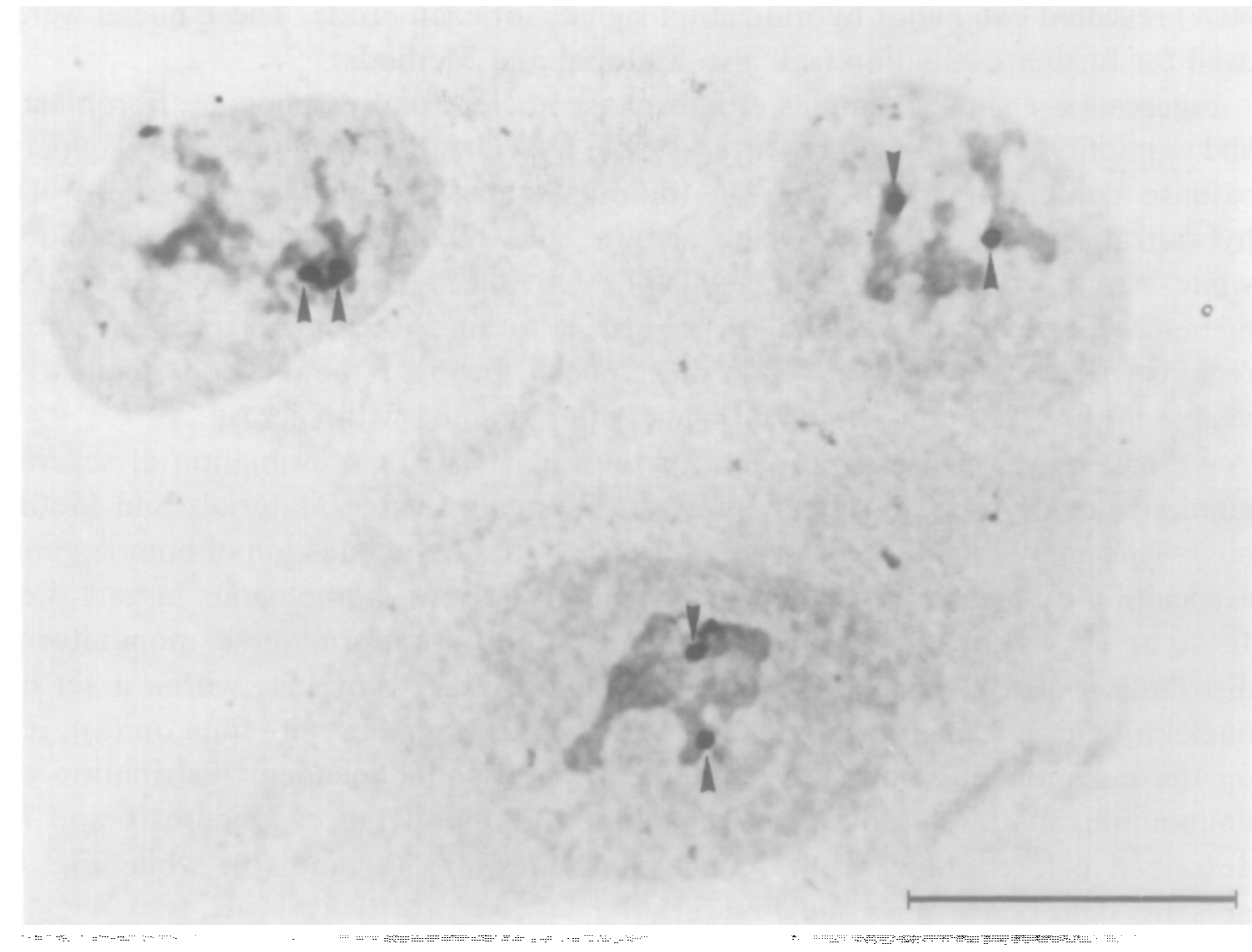

Fig. 5. Three typical nuclei from an amniotic fluid cell culture after fixation in situ, hybridization with biotinylated probe $15 \mathrm{c}$ (peroxidase detection), and Giemsa staining. Note the clear association of all hybridization signals (arrowheads) with the nucleoli. Bar represents $10 \mu \mathrm{m}$.
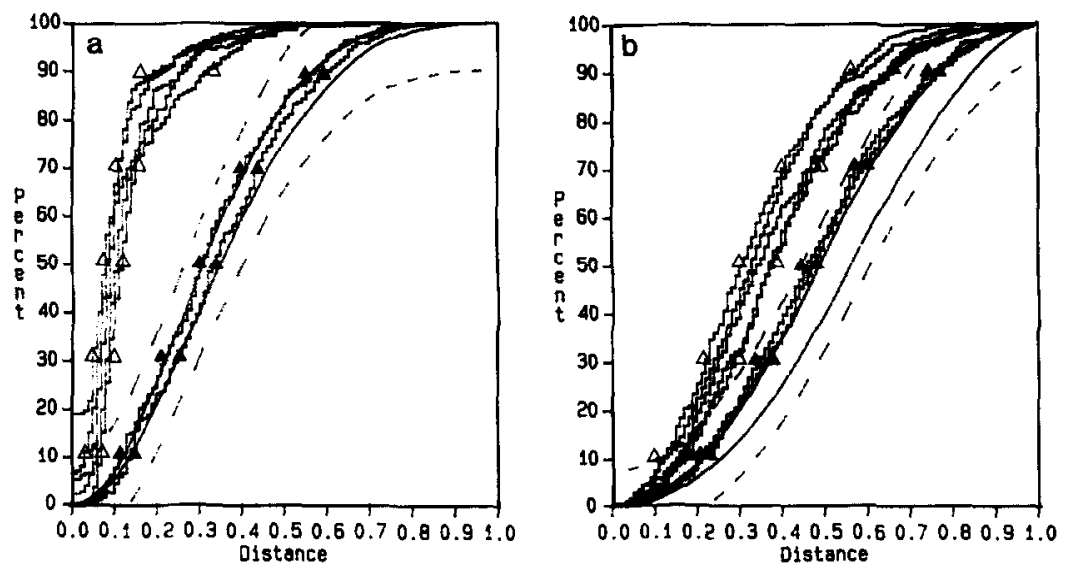

Fig. 6. Evaluation of target-target distances $(a)$ and target-center distances $(b)$ in human amniotic fluid cell nuclei after in situ hybridization with probe $15 \mathrm{c}(\Delta-\Delta)$ and/or probe 1c $(\Delta-\Delta)$. Data from five independent experiments are shown for probe $15 \mathrm{c}$. Three experiments were performed with probe $15 \mathrm{c}$ only (biotin/peroxidase detection). Double hybridization with probe $15 \mathrm{c}$ (biotin/alkaline phosphatase detection) and with probe $1 \mathrm{c}(\mathrm{Hg} /$ peroxidase detection) as carried out in two experiments. In each experiment, about 300 nuclei were evaluated. (Abscissa a) Normalized target-target distances between two hybridization spots on the two homologous chromosome regions. (Abscissa b) Normalized distances between one hybridization spot and the center of the nuclear image. (Ordinate) Percentage of nuclei with a normalized distance $\beta$ equal to or smaller than the corresponding $\beta$ shown on the abscissa. Two models for the random distribution of distances are indicated by smooth curves; hatched lines indicate $99 \%$ contidence limits. For details see legend of Fig. 2. 

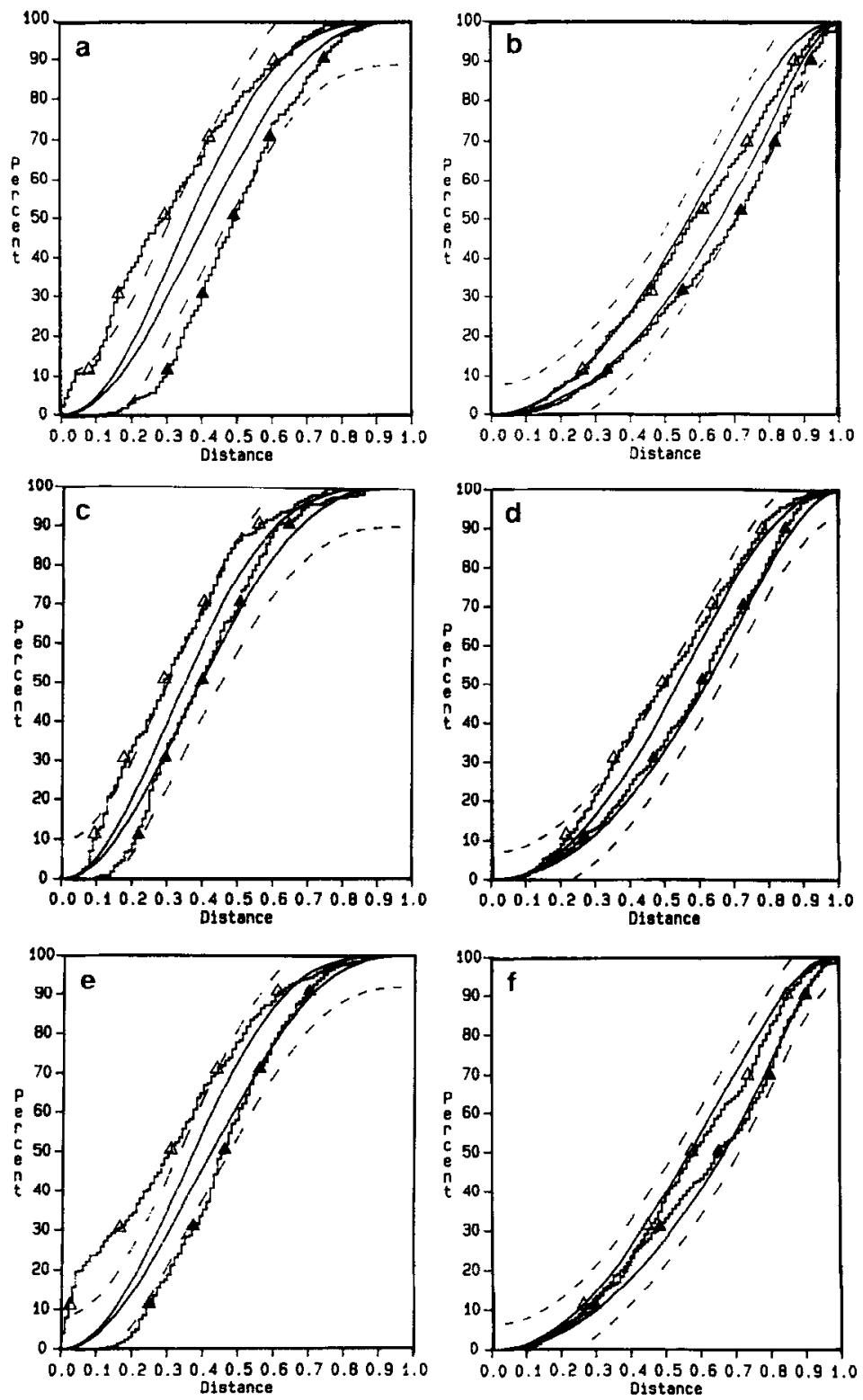

Fig. 7. Evaluation of target-target distances $(a, c, e)$ and target-center distances $(b, d, f)$ for probe $15 \mathrm{c}(\Delta-\Delta)$ and probe 1c $(\Delta-\Delta)$. Data in $(a-d)$ were obtained from single hybridization experiments with either probe 1c (biotin/peroxidase detection) or probe 15c (biotin/peroxidase detection). Data in $(e)$ and $(f)$ were obtained from double hybridization experiments with biotinylated probe 15c (alkaline phosphatase detection) and mercurated probe 1c (peroxidase detection). $(a, b)$ Evaluation of nonstimulated lymphocyte nuclei fixed without any pretreatment. $(c, d)$ Evaluation of lymphocyte nuclei fixed after PHA-stimulation of a blood culture for $72 \mathrm{~h}$, without colcemid and hypotonic treatment. $(e$, $f$ ) Evaluation of lymphocyte nuclei fixed after PHA-stimulation of a blood culture for $\mathbf{7 2} \mathrm{h}$, colcemid and hypotonic treatment. In each experiment, about 300 nuclei were evaluated. (Abscissa a, c, e) Normalized target-target distances between two hybridization spots on the two homologous chromosome regions. (Abscissa $b, d, f$ ) Normalized target-center distances. (Ordinate) Percentage of nuclei with a normalized distance $\beta$ equal to or smaller than the corresponding $\beta$ on the abscissa. Two models for the random distribution of distances are indicated by smooth curves; hatched lines indicate $99 \%$ confidence limits. For detail see legend of Fig. 2. 

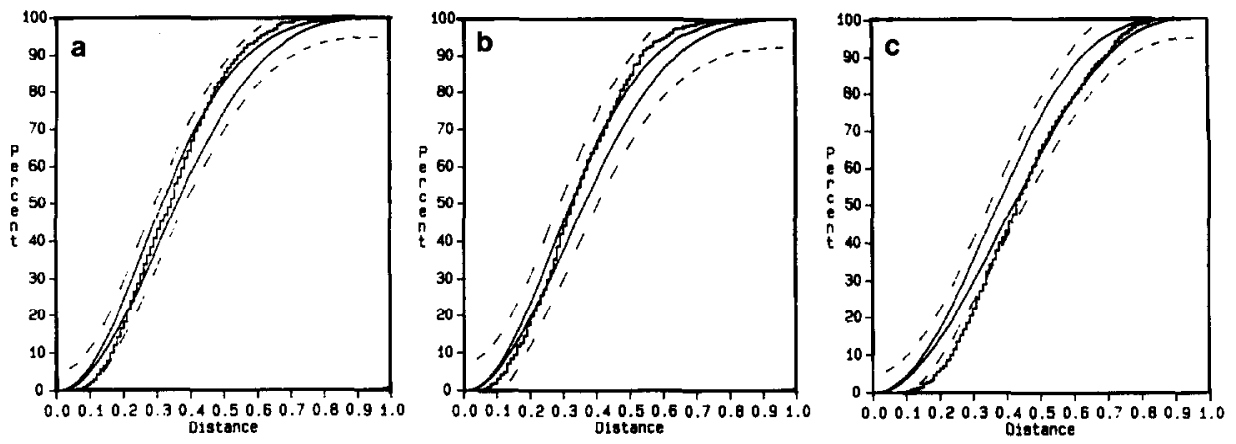

Fig. 8. Evaluation of target-target distances between hybridization spots on chromosome 1 and 15 after double hybridization with probe $1 \mathrm{c}(\mathrm{Hg} /$ peroxidase detection) and probe $15 \mathrm{c}$ (biotin/alkaline phosphatase detection. (a) Evaluation of human lymphocyte nuclei fixed after PHA-stimulation of a blood culture for $72 \mathrm{~h}$, colcemid and hypotonic treatment. (b) Evaluation of human amniotic fluid cell nuclei. (c) Evaluation of human fibroblast nuclei. Each curve represents data from about 600 nuclei pooled from two independent experiments. (Abscissa) Normalized distances between the hybridization spots on nonhomologous chromosomes. (Ordinate) Percentage of nuclei with a normalized distance $\beta$ equal or smaller to the corresponding $\beta$ on the abscissa. Two models for the random distribution of distances are indicated by smooth curves; hatched lines indicate $99 \%$ confidence limits. For details see legend of Fig. 2.

Figs. 6 and 7). In agreement with these other cell types, curves obtained for 1c-center distances (Figs. $7 b, d, f$ ) suggest a relatively uniform distribution of 1csites in human lymphocyte nuclei as well, while 1c-1c distances argue against homologous association (Figs. $7 a, c, e$ ). In fact, in two experiments (Figs. $7 a, e$ ) a slight excess of larger 1c-1c distances was observed. The possible effects of an additional colcemid and hypotonic shock treatment on $1 \mathrm{c}$ and $15 \mathrm{c}$ positioning in PHA-stimulated lymphocytes were small at best (compare Figs. $7 c$ and $d$ with $e$ and $f$ ). In our present experiments some $10-20 \%$ of nuclei in the evaluated areas exhibited only one single $1 \mathrm{c}$ or $15 \mathrm{c}$ spot. A subpopulation of cells with homologous association may be expected within this fraction of 1-spot nuclei. However, the size of this fraction has been largely preparation dependent suggesting insufficient probe penetration and/or loss of target DNA [7]. Accordingly, nuclei suspected of incomplete hybridization were excluded from further evaluation (see Material and Methods for complete selection criteria). In double hybridization experiments, an apparently close association of two 1c sites did not correlate with a predictable close association of $15 c$ sites and vice versa (see Fig. $4 a$ ). In conclusion, it appears that a subpopulation of cells which would generally exhibit close homologous association of $1 \mathrm{c}$ and $15 \mathrm{c}$ sites should be very small at best in the human cell types we have studied so far.

Figure 8 shows target-target distances between the heterologous $1 \mathrm{c}$ and $15 \mathrm{c}$ spots for PHA-stimulated lymphocytes fixed after colcemid and hypotonic treatment (Fig. 8a), amniotic fluid cells (Fig. 8b), and fibroblasts (Fig. 8c). In all three cell types a large variability of distances between the constitutive heterochromatin of the heterologous chromosomes 1 and 15 was observed. The curves for lymphocytes and amniotic fluid cells are located within range 2 (see Fig. 2), while 
in fibroblast nuclei a slight overrepresentation of larger distances was found. This has not been confirmed so far by independent experiments and thus may represent a statistical fluctuation.

For double hybridization experiments an additional evaluation was performed to prove or disprove the different distribution of $1 \mathrm{c}$ and $15 \mathrm{c}$ sites within a given set of nuclei. For each cell type the mean ratio of $1 \mathrm{c}-1 \mathrm{c}$ distances divided by $15 \mathrm{c}-15 \mathrm{c}$ distances as well as the mean ratio of $1 \mathrm{c}$-center distances divided by $15 c-c e n t e r$ distances was calculated (see Table 1). In all cases the mean ratios indicate significantly smaller distances for $15 \mathrm{c}$ spots than for $1 \mathrm{c}$ spots. Furthermore, a comparison of the deviations in the three different cell systems showed that they were similar for amniotic fluid cells and fibroblasts, as mentioned above, but were significantly smaller in stimulated lymphocytes than in amniotic fluid cells and fibroblasts ( $99 \%$ level as tested by Student's $t$ test).

\section{DISCUSSION}

In the present investigation, we have shown that the positions of the constitutive heterochromatin of the human chromosomes 1 and 15 can be simultaneously determined in interphase nuclei by double in situ hybridization with appropriate probes using different probe labeling (biotin, mercury) and probe detector systems (alkaline phosphatase, peroxidase, FITC, TRITC). In the following, we shall briefly discuss (1) particular advantages, limitations, and possible improvements of this approach and (2) biological implications of the findings obtained in the present investigation.

It is a particular advantage of double in situ hybridization experiments that a direct comparison of the distribution of different hybridization targets can be performed in each individual cell nucleus. In this way different distributions of these targets can be recognized even when the set of investigated nuclei is very heterogeneous in size and shape. In contrast, the comparison of the $2 \mathrm{D}$-distribution of distances obtained for two different probes in separate experiments is valid only if the two sets of evaluated nuclei are of identical 3D-configuration. From a different 2D-distribution a different 3D-distribution of hybridization targets can be deduced. Accordingly, we have obtained evidence for a distinctly different distribution of $1 \mathrm{c}$ and $15 \mathrm{c}$ sites in three different cell systems (lymphocytes, fibroblasts, and amniotic fluid cells). Furthermore, we were able to measure for the first time 2D-distances between nonhomologous chromosome segments in interphase nuclei and show that $1 \mathrm{c}$ and $15 \mathrm{c}$ sites were distributed independently from each other.

Of particular importance is the question whether in vivo arrangements of interphase chromosomes were sufficiently preserved in cells after fixation with methanol/acetic acid $(3: 1)$ and DNA denaturation. The 3D-structure of nuclei collapsed when fixed cells were air dried. The effect of such a collapse on 2Dmeasurements was likely to be more pronounced in spherical lymphocyte nuclei as compared to flat fibroblast nuclei [29], and in hypotonically swollen nuclei as compared to nonswollen nuclei. Although we expect that subtle arrangements of 


\section{Emmerich et al.}

chromatin fibers are affected by these treatments, the following observations argue against gross changes in the relative $2 \mathrm{D}$-positions of complete interphase chromosome domains as a result of our present fixation and hybridization procedures. First, in laser-uv-microirradiation experiments nuclei from living human and Chinese hamster fibroblastoid cells were microirradiated at one, two, or more defined positions [30, and unpublished data]. After fixation with methanol/ acetic acid $(3: 1)$ the microirradiated chromatin was visualized in air dried nuclei by indirect immunofluorescence using antibodies specific against uv-irradiated DNA. Under these conditions the irradiated chromatin remained at the expected sites. The relative distances between several irradiation sites were largely maintained even after hypotonic shock treatment. Second, Manuelidis [1, 31] has found no indication that the relative positions of structures such as nucleoli and clusters of heterochromatin in paraformaldehyde-fixed neuronal and glia cells were considerably changed by heat denaturation of nuclear DNA in the presence of formamide or by other steps involved in in situ hybridization of DNA probes and subsequent detection protocols. In the present experiments, 15c hybridization targets remained closely associated with nucleoli still reflecting the situation expected for these cells in vivo.

Possible improvements of this approach reflect the further development of fixation and denaturation procedures optimally suited to preserve the threedimensional morphology of interphase nuclei $[31,32]$ as well as the development of multi in situ hybridization protocols which allow the simultaneous visualization of more than two DNA target sites [33]. Combination of these improved techniques with light optical 3D-reconstruction of nuclei $[34,35,32]$ seems the most promising route for future studies of chromosome topography. Present procedures for the light optical 3D-reconstruction of nuclei using conventional light microscopy, however, are complicated and time consuming. Laser confocal scanning microscopy may provide a more convenient solution [36-39]. In contrast, the present approach based on 2D-analysis is simple, rapid, and it can easily be applied to screen large numbers of cell nuclei for nonrandom chromosome arrangements (see below). Based on this information, 3D-reconstruction may then be used to describe such arrangements, in a smaller set of nuclei in more detail.

In our present experiments, the distribution of $1 \mathrm{c}-1 \mathrm{c}$ and $1 \mathrm{c}-\mathrm{center}$ distances largely fits the random expectation, while $15 \mathrm{c}$ sites were distributed significantly closer to the center of the 2D-nuclear image in nuclei of human fibroblasts and amniotic fluid cells. Accordingly, $15 \mathrm{c}-15 \mathrm{c}$ distances were also significantly smaller. Similar, although less pronounced effects were observed in lymphocyte nuclei.

Our observation strongly argues against the idea of a close and permanent homologous association between the two 1c sites, although such an association cannot be ruled out for the other regions of this chromosome. In contrast, Hoehn and Martin [40] using the alkali-Giemsa method of Arrighi and Hsu [41] have suggested attraction between two lqh + regions in human fibroblast and amniotic fluid cell nuclei which ultimately may result in apparent fusion. 
While most $15 \mathrm{c}$-spots were closely associated with the nucleoli, their arrangements in individual nuclei appeared otherwise quite independent. In cells with several nucleoli, 15c spots were found to be associated with these nucleoli in any possible combination, i.e., with different nucleoli or with one nucleolus. In the latter case, 15c spots were observed close to each other or separated from each other at varying degrees up to an opposite position. In conclusion, we feel that the smaller distances between $15 \mathrm{c}$ spots are likely derived by their nucleolar associations and not by homologous association per se.

Data published on the question of homologous association for other mammalian cells using other methods are contradictory. In agreement with this investigation laser uv-microirradiation experiments also argue against the close and permanent association of homologous chromosomes in fibroblastoid Chinese hamster cells [42, 43]. In contrast Hadlaczky et al. [44] reported that homologous chromosomes recognized by immunostained centromeres occupy adjacent territories in $30 \%$ of Indian muntjac cells in which centromeres of individual chromosomes could be identified. Differences in the methods applied in these investigations do not suffice in our opinion to explain these and other findings (see also below).

Our data are also in conflict with reports which indicate the mutually dependent distribution of 1q12 and NOR-bearing chromosomes in both human lymphocytes [8, 9, 15] and fibroblasts [16]. Recently, Manuelidis and Borden [32] have found in large human neurons that one 1q12 region was always compartmentalized together with centromeres on the nucleolus, while the other 1q12 region either abutted the nucleolus or was on the nuclear membrane. In astrocytes, however, these chromosome subregions were always associated with the nuclear membrane and not necessarily with nucleoli.

These findings reinforce the view that chromosome positioning may be cell type specific. Apparent contradictions of the findings reported in the literature may then reflect the possibility that this positioning is not rigid but dynamic even in highly differentiated cells [31] and may reflect the physiological state of a given cell population [2].

We thank Dr. H. Cooke for a gift of pUC.177, Drs. M. Schardin, P. Lichter, and L. Manuelidis for helpful comments on the manuscript, and Dr. W. Schlegel for stimulating discussions of image analysis problems and for the possibility to use the VAX 11-780 computer of the Institute of Nuclear Medicine at the German Cancer Research Center in Heidelberg. We are especially grateful to Dipl. Math. J. Krüger for his advice in the statistical analysis of the data. This work was supported by a Heisenbergstipendium of the Deutsche Forschungsgemeinschaft for T. Cremer and by a Biostipenlium of the Bundesministerium für Forschung und Technologie for P. Emmerich and A. Jauch.

\section{REFERENCES}

1. Manuelidis, L. (1984) Proc. Natl. Acad. Sci. USA 81, 3123-3127.

2. Blobel, G. (1985) Proc. Natl. Acad. Sci. USA 82, 8527-8529.

3. Gasser, S. M., and Laemmli, U. K. (1987) Trends Genet. 3, 16-22.

4. Cremer, T., Emmerich, P., and Lichter, P. (1987) Ann. Univ. Sarav. Med. (Suppl.) 7, 67-71.

5. Cooke, H. J., and Hindley, J. (1979) Nucleic Acids Res. 10, 3177-3197.

5. Higgins, M. J., Wang, H., Shtromas, J., Haliotis, T., Roder, J. C., Holden, J., and White, B. N. (1985) Chromosoma 93, 77-86. 
7. Cremer, T., Tesin, D., Hopman, A. H. N., and Manuelidis, L. (1988) Exp. Cell Res. 126, 199-220.

8. Chandra, H. S., Narayana, A. K. A., Bucke, V. V., and Hungerford, D. A. (1972) J. Genet. Hum. 61, 78-83.

9. Galperin-Lemaitre, H., Hens, L., Kirsch-Volders, M., and Susanne, C. (1977) Hum. Genet. 35, 261-268.

10. Sinkus, A., Wachtler, F., and Schwarzacher, H. G. (1980) Hum. Genet. 54, 125-126.

11. Hager, H. D., Schroeder-Kurth, T. M., and Vogel, F. (1982) Hum. Genet. 61, 341-356.

12. Ockey, C. H. (1969) Chromosoma 27, 308-320.

13. Rodman, T. C., Flehinger, B. G., and Squire, R. D. (1978) Hum. Genet. 41, 19-34.

14. Wollenberg, C., Kiefaber, M. P., and Zang, K. D. (1982) Hum. Genet. 62, 310-315.

15. Schmid, M., Vogel, W., and Krone, W. (1975) Cytogenet. Cell Genet. 15, 66-80.

16. Stahl, A., Hartung, M., Vagener-Capodana, M., and Fouet, C. (1976) Hum. Genet. 35, 27-34.

17. Ochs, B. A., Franke, W. W., Moll, R., Grund, C., Cremer, M., and Cremer, T. (1983) Differentiation 24, 153-173.

18. Maniatis, T., Fritsch, E. F., and Sambrook, J. (1982) Molecular Cloning: A Laboratory Manual, Cold Spring Harbor Laboratory, Cold Spring Harbor, NY.

19. Langer, P. R., Waldrop, A. A., and Ward, D. C. (1981) Proc. Natl. Acad. Sci. USA 78, $6633-6637$.

20. Dale, R. M. K., Martin, E., Ljvingston, D. C., and Ward, D. C. (1975) Biochemistry 14, 2447-2457.

21. Hopman, A. H. N., Wiegant, J., and v. Duijn, P. (1986) Nucleic Acids Res. 14, 6471-6488.

22. Schardin, M., Cremer, T., Hager, H. D., and Lang, M. (1985) Hum. Genet. 71, 281-287.

23. Hopman, A. H. N., Wiegant, J., and v. Duijn, P. (1987) Exp. Cell Res. 169, 357-368.

24. Hopman, A. H. N., Wiegant, J., Raap, A. K., Landegent, J. E., v. d. Ploeg, M., and v. Duijn, P. (1986) Histochemistry 85, 1-4.

25. Loos, P. (1987) Diplomarbeit, Fakultät für Physik und Astronomie, Universität Heidelberg.

26. Zinser, G., and Komitowski, D. (1982) J. Histochem. Cytochem. 31, 94-100.

27. Rappold, G. A., Cremer, T., Hager, H. D., Davies, K. E., Müller, C. R., and Yang, T. (1984) Hum. Genet. 67, 317-325.

28. Sachs, L. (1968) Angewandte Statistik. Springer Verlag, Berlin, New York.

29. Bourgeois, C. A., Hemon, D., Beaure D'Augeres, C., Robineuax, R., and Boutielle, M. (1981) Biol. Cell 40, 229-232.

30. Cremer, T., Baumann, H., Nakanishi, K., and Cremer, C. (1984) in Chromosomes Today (Bennett, M. D., Gropp, A., and Wolf, U., Eds.), Vol. 8, pp. 203-212, Allen \& Unwin, London.

31. Manuelidis, L. (1984) J. Neuropathol. Exp. Neurol. 43, 225-241.

32. Manuelidis, L., and Borden, J. (1988) Chromosoma 96, 397-410.

33. Nederlof, P. M., Raap, A. K., Robinson, D., Wiegant, J., Hopman, A. H. N., and Tanke, H. J. (1987) Eur. J. Cell Biol. 44, (Suppl 20), 47. [Abstract]

34. Erhardt, A., Zinser, G., Komitowski, D., and Bille, J. (1985) Appl. Optics 24, 194-200.

35. Pinkel, D., Gray, J. W., Trask, B., v. d. Engh, G., Fuscoe, J., and v. Dekken, H. (1986) Cold Spring Harbor Symp. Quant. Biol. 51, 151-157.

36. Cremer, C., and Cremer, T. (1978) Microsc. Acta 81, 31-44.

37. Brakenhoff, G. J., v. d. Voort, H. T. M., v. Spronsen, E. A., Linnemans, W. A. M., and Nanninga, N. (1985) Nature (London) 317, 748-749.

38. Wijnaendts van Resandt, R. W., Marsman, H. J. B., Kaplan, R., Davoust, J., Stelzer, E. H. K., and Striker, R. (1985) J. Microsc. B8, 29-34.

39. White, J. G., Amos, W. B., and Fordham, M. (1987) J. Cell Biol. 105, 41-48.

40. Hoehn, H., and Martin, G. M. (1973) Cytogenet. Cell Genet. 12, 443-452.

41. Arrighi, F. E., and Hsu, T. C. (1971) Cytogenetics 10, 81-86.

42. Cremer, T., Cremer, C., Baumann, H., Lüdtke, E. K., Sperling, K., Teuber, V., and Zorn, G. (1982) Hum. Genet. 60, 46-56.

43. Cremer, T., Cremer, C., Schneider, T., Baumann, H., Hens, L., and Kirsch-Volders, M. (1982) Hum. Genet. 62, 201-209.

44. Hadlaczky, G. Y., Went, M., and Ringertz, N. R. (1986) Exp. Cell Res. 167, 1-15.

Received June 8, 1988 